\title{
Mining the potential prognostic value of synaptosomal- associated protein 25 (SNAP25) in colon cancer based on stromal-immune score
}

\author{
Jinyan Zou ${ }^{1}$, Darong Duan ${ }^{2}$, Changfa Yu ${ }^{2}$, Jie Pan ${ }^{3}$, Jinwei Xia ${ }^{2}$, Zaixing Yang $^{2}$, Shasha Cai ${ }^{\text {Corresp. }}{ }^{2}$ \\ ${ }^{1}$ Department of Gastroenterology, Taizhou First People's Hospital, Huangyan Hospital of Wenzhou Medical University, Zhejiang, China, Taizhou, Zhejiang, \\ China \\ 2 Department of Laboratory Medicine, Taizhou First People's Hospital, Huangyan Hospital of Wenzhou Medical University, Zhejiang, China, Taizhou, \\ Zhejiang, China \\ 3 Outpatient Department, Taizhou First People's Hospital, Huangyan Hospital of Wenzhou Medical University, Zhejiang, China, Taizhou, Zhejiang, China \\ Corresponding Author: Shasha Cai \\ Email address: tyycss@163.com
}

Background: Colon cancer is one of the deadliest tumors worldwide. Stromal cells and immune cells play important roles in cancer biology and microenvironment across different types of cancer. This study aimed to identify the prognostic value of stromal/immune cellassociated genes for colon cancer in The Cancer Genome Atlas (TCGA) database using bioinformatic technology. Methods: The gene expression data and corresponding clinical information of colon cancer were downloaded from TCGA database. Stromal and immune scores were estimated based on the ESTIMATE algorithm. Sanger software was used to identify the differentially expressed genes (DEGs) and prognostic DEGs based on stromal and immune scores. External validation of prognostic biomarkers was conducted in Gene Expression Omnibus (GEO) database. Gene ontology (GO) analysis, pathway enrichment analysis, and gene set enrichment analysis (GSEA) were used for functional analysis. STRING and Cytoscape were used to assess Protein-protein interaction (PPI) network and screen hub genes. Quantitative real-time PCR (qRT-PCR) was used to validate the expression of hub genes in clinical tissues. Synaptosomal-associated protein 25 (SNAP25) was selected for analyzing its correlations with tumor-immune system in TISIDB database. Results: Worse overall survivals of colon cancer patients were found in high stromal score group (2963 vs. 1930 days, log-rank test $P=0.038$ ) and high immune score group (2894 vs. 2230 days, log-rank test $P=0.076) .563$ up-regulated and 9 down-regulated genes were identified as stromal-immune score-related DEGs. 70 up-regulated DEGs associated with poor outcomes were identified by COX proportional hazard regression model, and 15 hub genes were selected later. Then, we verified aquaporin 4 (AQP4) and SNAP2 5 as prognostic biomarkers in GEO database. qRT-PCR results revealed that AQP4 and SNAP25 were significantly elevated in colon cancer tissues compared with adjacent normal tissues 
$(P=0.003,0.001)$. GSEA and TISIDB suggested that SNAP25 involved in cancer-related signaling pathway, immunity and metabolism progresses. Conclusion: SNAP25 is a microenvironment-related and immune-related gene that can predict poor outcomes in colon cancer. 


\section{Mining the potential prognostic value of synaptosomal-associated protein 25 (SNAP25) in colon cancer based on stromal-immune score}

3 Jinyan Zou ${ }^{1}$, Darong Duan ${ }^{2}$, Changfa $\mathrm{Yu}^{2}$, Jie Pan ${ }^{3}$, Jinwei $\mathrm{Xia}^{2}$, Zaixing Yang ${ }^{2}$, Shasha Cai ${ }^{2}$

$4{ }^{1}$ Department of Gastroenterology, Taizhou First People's Hospital, Huangyan Hospital of

5 Wenzhou Medical University, Zhejiang, China

6 'Department of Laboratory Medicine, Taizhou First People's Hospital, Huangyan Hospital of

7 Wenzhou Medical University, Zhejiang, China

$8{ }^{3}$ Outpatient Department, Taizhou First People's Hospital, Huangyan Hospital of Wenzhou

9 Medical University, Zhejiang, China.

10 Corresponding Author:

11 Zaixing Yang ${ }^{2}$

12 No 218 Hengjie Road, Huangyan District, Taizhou City, Zhejiang Province, 318020, China

13 E-mail: yangzaixingdiyi@163.com

14 Shasha $\mathrm{Cai}^{2}$

15 No 218 Hengjie Road, Huangyan District, Taizhou City, Zhejiang Province, 318020, China

16 E-mail: tyycss@163.com

\section{Abstract}

Background: Colon cancer is one of the deadliest tumors worldwide. Stromal cells and immune cells play important roles in cancer biology and microenvironment across different types of cancer. This study aimed to identify the prognostic value of stromal/immune cell-associated genes for colon cancer in The Cancer Genome Atlas (TCGA) database using bioinformatic technology.

Methods: The gene expression data and corresponding clinical information of colon cancer were downloaded from TCGA database. Stromal and immune scores were estimated based on the ESTIMATE algorithm. Sanger software was used to identify the differentially expressed genes (DEGs) and prognostic DEGs based on stromal and immune scores. External validation of prognostic biomarkers was conducted in Gene Expression Omnibus (GEO) database. Gene ontology (GO) analysis, pathway enrichment analysis, and gene set enrichment analysis (GSEA) were used for functional analysis. STRING and Cytoscape were used to assess Protein-protein interaction (PPI) network and screen hub genes. Quantitative real-time PCR (qRT-PCR) was used to validate the expression of hub genes in clinical tissues. Synaptosomal-associated protein 25 (SNAP25) was selected for analyzing its correlations with tumor-immune system in TISIDB database. 
35 Results: Worse overall survivals of colon cancer patients were found in high stromal score group 36 (2963 vs. 1930 days, log-rank test $\mathrm{P}=0.038)$ and high immune score group (2894 vs. 2230 days,

37 log-rank test $\mathrm{P}=0.076$ ). 563 up-regulated and 9 down-regulated genes were identified as stromal38 immune score-related DEGs. 70 up-regulated DEGs associated with poor outcomes were 39 identified by COX proportional hazard regression model, and 15 hub genes were selected later. 40 Then, we verified aquaporin 4 (AQP4) and SNAP25 as prognostic biomarkers in GEO database. 41 qRT-PCR results revealed that AQP4 and SNAP25 were significantly elevated in colon cancer 42 tissues compared with adjacent normal tissues $(\mathrm{P}=0.003,0.001)$. GSEA and TISIDB suggested 43 that SNAP25 involved in cancer-related signaling pathway, immunity and metabolism 44 progresses.

45 Conclusion: SNAP25 is a microenvironment-related and immune-related gene that can predict 46 poor outcomes in colon cancer.

47 Keywords: colon cancer, stromal, immune, TCGA, GEO, prognosis

48 Introduction

49 Colon cancer is the third most common malignant tumor and one of the leading causes of 50 cancer-related death worldwide. Since risk factors have been investigated, earlier detection, 51 cancer prevention, surgical techniques, radiotherapy and chemotherapy treatment have been improved, the incidence and mortality of colon cancer has been slowly declined. A model-based estimate showed that 104610 new cases of colon cancer would be diagnosed and 53200 patients would die of colon cancer in United States (Siegel et al. 2020).However, the incidence of colorectal cancer in patients aged $<50$ years increased by $22 \%$ from 2000-2013(Siegel et al. _ 2017). Thus, more attention should be focused on early diagnosis through screening and accurately predicting the survival outcome of patients with colon cancer.

Stromal cells and immune cells form the major fraction of colon cancer tissue and are associated with tumor progress, inflammatory and metabolic disorders(Ghesquière et al. 2014; Nilendu et al. 2018). An increasing amount of studies have highlighted the importance of stromal cells and immune cells in cancer biology and microenvironment across different types of cancers(Barros et al. 2018; Garcia-Gomez et al. 2018; Zhan et al. 2017). ESTIMATE is a new method that infers stromal and immune cells in malignant tumors using gene expression signatures(Yoshihara et al. 2013), and has been conducted in acute myeloid leukemia(Yan et al. 2019), gastric cancer(Wang et al. 2019), and glioblastoma(Jia et al. 2018). 
66

67

68

69

70

71

72

73

74

75

76

77

78

79

80

81

82

83

84

85

86

87

88

89

90

91

92

93

94

95

In the current study, we obtained immune and stromal scores of colon cancer based on ESTIMATE. To help elucidate the stromal-immune score-based genes with prognostic value in colon cancer, we obtained gene expression dates from The Cancer Genome Atlas (TCGA), and verified the survival value in a different colon cancer cohort available from the Gene Expression Omnibus (GEO) database. Two hub genes were validated to be prognosis biomarkers and selected for further analysis. We investigated the potential underlying mechanisms of synaptosomal-associated protein 25 (SNAP25) in cancer-related signaling pathway, immunity and metabolism progresses through gene set enrichment analysis (GSEA) and TISIDB database.

\section{Materials \& Methods}

\section{Database and estimation of stromal and immune scores}

The TCGA level 3 gene expression data and corresponding clinical information for colon cancer patients were obtained from Genomic Date Commons of the National Cancer Institute (http://portal.gdc.cancer.gov). Only patients with gene expression data, follow up information and clinicopathologic information were included in this study. For normalization, the RNA-seq data of all patients was transformed to transcripts per million (TPM) values (https://pubmed.ncbi.nlm.nih.gov/30379987/). The stromal and immune scores for each TCGA sample were conducted by R 3.6.2 using the R package "estimate" (https://pubmed.ncbi.nlm.nih.gov/24113773/).

We obtained gene expression profiles and clinical information of 430 colon cancer patients from TCGA database. Among them, 231 (53.7\%) cases were male and 199 (46.3\%) cases were female. The average age of patients at initial pathological diagnosis was 66.3 years (range: $31-90$ years). Histologic diagnosis included 369 (85.5\%) cases of colon adenocarcinoma and 57 $(13.3 \%)$ cases of colon mucinous adenocarcinoma, 4 (0.9\%) cases were not classified. The tumor stage was stage I in $17.4 \%$, stage II in $38.6 \%$, stage III in 29.1 , stage IV in $14.4 \%$ of cases, and 2 cases $(0.5 \%)$ were of unknown stage. The tumors were located in the left $(40 \%)$ or right $(55.3 \%)$ colon according to their anatomic neoplasm subdivision, with 4.7\% unknown. Based on ESTIMATE algorithm, we obtained stromal scores (range: -2262.07 1999.52) and immune scores (range: -954.97 3035.59) for all these colon cancer patients.

\section{Correlations between clinicopathologic data and stromal/immune score}


96

97

98

99

100

101

102

103

104

105

106

107

108

109

110

111

112

113

114

115

116

117

118

119

120

121

122

123

124

125

126

The correlations between clinicopathologic data and stromal/immune score were analyzed by SPSS 22.0 software (SPSS, Inc., Chicago, IL, USA). Patients with colon cancer were divided into high stromal/immune score (the fourth quartile) and low stromal/immune score groups (quartile 1-3). The stromal/immune score of different clinicopathologic groups was compared by Mann-Whitney U test, and overall survival was estimated by the Kaplan-Meier method and compared by log-rank tests. A value of $\mathrm{P}<0.05$ was considered statistically significant.

\section{Identification of differentially expressed genes (DEGs)}

DEGs were identified based on immune and stromal scores (high stromal/immune score group vs. low stromal/immune score group) by Sanger_V1.0.8 software (https://shengxin.ren/ ). Genes with $\log 2$ (fold change) $>1.5$ or $<-1$ combined with a $\mathrm{P}$ value $<0.01$ were defined as DEGs. The volcano plot of the DEGs was drawn by Sanger_V1.0.8 software, and the venn diagram was drawn on a website Venny 2.1.0

(https://bioinfogp.cnb.csic.es/tools/venny/index.html).

\section{Gene ontology (GO) and pathway enrichment analyses}

Cellular component (CC), molecular function (MF), biological process (BP) and pathway enrichment analyses were conducted using FunRich 3.1.3

(https://pubmed.ncbi.nlm.nih.gov/25921073/). A value of $\mathrm{P}<0.05$ was considered as the screening condition.

\section{Survival analysis}

A COX proportional hazards model was applied to illuminate prognostic DEGs of colon cancer obtained from TCGA and a P value $<0.01$ was considered significant. An open source web tool PrognoScan (https://pubmed.ncbi.nlm.nih.gov/19393097/) was conducted to verify the survival outcomes between prognostic DEGs identified and colon cancer patients from GEO database.

\section{Protein-protein interaction (PPI) network construction}

The PPI network for DEG-encoded proteins was performed by STRING database (https://pubmed.ncbi.nlm.nih.gov/30476243/) and reconstructed by Cytoscape 3.7.2 (https://pubmed.ncbi.nlm.nih.gov/14597658/). The most significant modular analysis was identified by Molecular Complex Detection (MCODE) plugin of Cytoscape, and the plug-in Biological Networks Gene Oncology (BiNGO) of Cytoscape was applied to analysis GO term of hub genes. 


\section{Heatmap and clustering analysis}

128

129

130

131

132

133

134

135

136

137

138

139

140

141

142

143

144

145

146

147

148

149

150

151

152

153

154

155

156

157

Heatmap and clustering analysis were completed by "heatmap" package.

\section{Quantitative real-time PCR (qRT-PCR)}

Total RNA was extracted with Trizol reagent (TaKaRa Bio Inc. Shiga, Japan) from colon cancer and adjacent normal tissues. cDNA was synthesized with PrimeScript ${ }^{\mathrm{TM}} \mathrm{RT}$ reagent kit (TaKaRa, RR036A). Quantitative real-time PCR (qRT-PCR) was carried out using the TB Green $^{\mathrm{TM}}$ Premix Ex Taq ${ }^{\mathrm{TM}}$ kit (TaKaRa, RR420A) on ABI step one Real-Time PCR system. The primers were as follows: AQP4, sense strand 5'- GAGCAGGAATCCTCTATC-3', antisense strand 5'- AGTGACATCAGTCCGTTT-3'; SNAP25, sense strand 5'-

GTAGTGGACGAACGGGAGC-3', antisense strand 5'- CCTGTCGATCTGGCGATT-3'; GAPDH, sense strand 5'-GTCAACGGATTTGGTCTGTATT-3', antisense strand 5'-

AGTCTTCTGGGTGGCAGTGAT-3'.

The Institutional Medical Ethics Review Board of Taizhou first people's hospital in Zhejiang Province approval to carry out the study within its facilities (Ethical Application Ref: 2019-KY009-03).

\section{Gene set enrichment analysis (GSEA)}

According to the expression level of AQP4 (or SNAP25), samples of the complete cohort from TCGA were divided into 2 groups, and implemented using GSEA by Sanger_V1.0.8 software. The KEGG gene set biological process database (c2.cp.kegg.v6) were chosen for enrichment analysis. Terms with both $\mathrm{P}$ value $<0.01$ and false discovery rate $(\mathrm{FDR})<0.01$ were identified.

\section{Mining the immune-related mechanism of SNAP25.}

TISIDB (https://pubmed.ncbi.nlm.nih.gov/14597658/) is a friendly web portal integrates 988 immune-related anti-tumor genes from 7 databases(Ru et al. 2019). The correlations between immune features and any gene may be explored in 30 TCGA cancer types. In this study, the TISIDB database was used to investigate the associations between expression (or methylation) of SNAP25 and tumor-infiltrating lymphocytes (TILs). However, the relations between TILs and expression (or methylation) of AQP4 in colon cancer were not integrated in TISIDB database. A value of $\mathrm{P}<0.05$ was considered statistically significant.

\section{Results}


158 The relationships between stromal/immune score and clinical features

159 The stromal and immune scores were variously distributed between adenocarcinoma and 160 mucinous adenocarcinoma (Figure 1A, C). Both stromal score and immune score of colon 161 adenocarcinoma cases were significantly lower than those of mucinous adenocarcinoma cases $162(\mathrm{P}=0.001,0.011$, respectively). In addition, right colon tumors yielded higher immune scores 163 than those left colon cases (Figure 1D, $\mathrm{P}<0.001$ ), though no significant differences between left 164 and right colon were found for the stromal scores (Figure 1B, $\mathrm{P}=0.818$ ).

165 The potential association of overall survival and stromal/immune score was explored by 166 classifying 430 colon cancer patients into high and low score groups based on their stromal or 167 immune scores. As shown in Figure 1E, F, the median overall survival of patients with a low 168 stromal score was longer than those in high score group (2963 vs. 1930 days, log-rank test $169 \mathrm{P}=0.038$ ); consistently, the median overall survival of patients with a low immune score was 170 longer than those in high score group ( 2894 vs. 2230 days, log-rank test $\mathrm{P}=0.076$ ), although there 171 was no statistically significant difference. However, patients with both a high stromal score and a 172 high immune score were found to have significantly worse survival than those with low scores 173 (1891 vs. 2974 days, log-rank test $\mathrm{P}=0.039$ ) (Figure $1 \mathrm{G}$ ).

\section{Identification of DEGs by stromal and immune scores in colon cancer}

175 After standardization of the RNA-Seq data for all 430 colon cancer patients obtained from 176 TCGA database, we identified 4881 and 1512 DEGs based on stromal and immune scores, 177 respectively. As shown in the volcano plots of DEGs for stromal/immune score (Figure 2A, B), 1784791 genes were up-regulated and 90 genes were down-regulated for the comparison based on 179 stromal score. Similarly, 1113 genes were up-regulated and 399 genes were down-regulated 180 based on high immune score group vs. low immune score group. Through Venn diagram (Figure 181 2C, D) analysis, 563 shared up-regulated DEGs and 9 shared down-regulated DEGs from 182 stromal score and immune score groups were identified and selected for subsequent analysis.

\section{Functional and pathway enrichment analyses}

Go and pathway enrichment analyses of the above 572 genes were performed (Figure 2E, F, G, H). For CC, DEGs were mainly associated with plasma membrane, extracellular and extracellular space. With regard to MF, genes were mainly clustered in receptor activity, cell adhesion molecule activity and B cell receptor activity. DEGs in the BP category primarily enriched in immune response, cell communication and signal transduction. The pathway 
189

190

191

192

193

194

195

196

197

198

199

200

201

202

203

204

205

206

207

208

209

210

211

212

213

214

215

216

217

218

219

enrichment analysis showed genes were mainly enriched in epithelial-to-mesenchymal transition, peptide ligand-binding receptors and GPCR ligand binding.

\section{Identification of prognostic DEGs in colon caner}

The COX proportional hazard regression model was constructed to identify potential prognostic DEGs in colon cancer. Among the 563 shared up-regulated DEGs and 9 shared downregulated DEGs, 70 up-regulated DEGs associated with poor outcomes were shown in Figure 3.

\section{PPI network construction and hub gene analysis among prognostic DEGs}

To further explore the interplay among the 70 identified prognostic DEGs, we conducted a PPI network containing 30 nodes and 53 edges based on STRING tool and Cytoscape software (Figure 4A). Module analysis using MCODE was constructed, and 15 hub genes were selected (Figure 4A). CC, MF, BP analyses of the total 15 hub genes were performed using BiNGO (Figure 4B). The 15 hub genes were mainly associated with plasma membrane, transporter activity, secretion and channel activity.

\section{Heatmap and clustering analysis of 15 hub genes}

The expression level of 15 hub genes in "dead" and "alive" groups was shown in Figure 4C.

\section{Verifying the survival outcomes of hub genes in the GEO database}

The survival outcomes of 15 hub genes were identified on the PrognoScan web tool, which provided overall survival of GSE12945, GSE17536 and GSE17537 datasets for colorectal cancer. Then, AQP4 and SNAP25 were verified (Figure 5) to be significantly associated with overall survival according to both the log-rank test and COX proportional hazards regression analysis (all $\mathrm{P}<0.05)$.

\section{The expression of two hub genes in $\mathbf{2 0}$ colon cancer and adjacent normal tissue sample set} using qRT-PCR.

The expression of AQP4 and SNAP25 was validated in 20 pairs of clinical tissues using qRT-PCR. Interestingly, the mRNA relative expression levels of both AQP4 and SNAP25 were significantly elevated in colon cancer tissues compared with adjacent normal tissues $(\mathrm{P}=0.003$, 0.001) (Figure 6).

\section{GSEA using TCGA database}

To further investigate the underlying mechanism of AQP4 and SNAP25 in colon cancer, KEGG pathway enrichment analysis was performed by GSEA. For AQP4, "vascular smooth muscle contraction" (NES=2.17, $\mathrm{P}<0.001, \mathrm{FDR}=0.006)$ gene set was prominently enriched. For 
220 SNAP25, 57 gene sets were enriched, including 11 gene sets which were cancer-related 221 processes (Figure 7). Besides, high expression of SNAP25 might also be involved in "B cell 222 receptor signaling pathway" $(\mathrm{NES}=2.06, \mathrm{P}=0.002, \mathrm{FDR}=0.002)$, "cell adhesion molecules cams" 223 (NES=2.05, $\mathrm{P}=0.008, \mathrm{FDR}=0.002)$, "chemokine signaling pathway" ( $\mathrm{NES}=2.07, \mathrm{P}=0.002$, 224 FDR=0.002), "complement and coagulation cascades" (NES=2.08, $\mathrm{P}<0.001, \mathrm{FDR}=0.002)$, "T 225 cell receptor signaling pathway" ( $\mathrm{NES}=2.06, \mathrm{P}<0.001, \mathrm{FDR}=0.002)$, "adipocytokine signaling 226 pathway" ( $\mathrm{NES}=1.96, \mathrm{P}<0.001, \mathrm{FDR}=0.005)$, "aldosterone regulated sodium reabsorption" 227 (NES=2.02, $\mathrm{P}<0.001, \mathrm{FDR}=0.003$ ), "glycosaminoglycan biosynthesis heparan sulfate" 228 (NES=2.00, $\mathrm{P}<0.001, \mathrm{FDR}=0.004$ ), and "insulin signaling pathway" ( $\mathrm{NES}=1.94, \mathrm{P}=0.002$, $229 \mathrm{FDR}=0.007)$. This suggested that immunity and metabolism may be as well involved in the 230 underlying mechanism of SNAP25 in colon cancer.

231

232

233

234

235

236

237

238

239

240

241

242

243

244

245

246

247 248

249

250

\section{Regulation of immune molecules by SNAP25}

The spearman's correlations between lymphocytes and expression, methylation of SNAP25 were performed in TISIDB database (Figure 8). The associations between the expression of SNAP25 and immune-related signatures of TILs types were shown in Figure 8A, and the greatest correlations including natural killer cell (NK; $\mathrm{r}=0.493, \mathrm{P}<2.2 \mathrm{e}-16)$, macrophage $(\mathrm{r}=0.45, \mathrm{P}<2.2 \mathrm{e}-$ 16), mast cell ( $\mathrm{r}=0.448, \mathrm{P}<2.2 \mathrm{e}-16$ ), and natural killer $\mathrm{T}$ cell (NKT; $\mathrm{r}=0.447, \mathrm{P}<2.2 \mathrm{e}-16$ ) were shown in Figure 8B. The correlations between methylation of SNAP25 and lymphocytes were shown in Figure 8C, and Figure 8D displayed the remarkable negative correlations including plasmacytoid dendritic cell ( $\mathrm{pDC}$; $\mathrm{r}=-0.419, \mathrm{P}=2.96 \mathrm{e}-14)$, type $1 \mathrm{~T}$ helper cell (Th1; $\mathrm{r}=-0.406$, $\mathrm{P}=4.06 \mathrm{e}-13)$, $\mathrm{T}$ follicular helper cell (Tfh; $\mathrm{r}=-0.391, \mathrm{P}=3.78 \mathrm{e}-12)$, and NKT ( $\mathrm{r}=-0.391, \mathrm{P}=3.61 \mathrm{e}-$

12). Therefore, the potential underlying mechanism of SNAP25 in colon cancer may be involved in the regulation of the above TILs.

\section{Discussion}

Solid tumor tissues comprise not only malignant cells but also tumor microenvironment, including immune cells, stromal cells, epithelial cells, fibroblasts, vascular cells and signaling molecules. Accumulating evidence clarifies that tumor microenvironment plays a crucial role in tumor growth, progression, metastasis, prognosis, and treatment(Petitprez et al. 2018; Wu \& Dai 2017). In the current study, we focused on stromal and immune scores, which reflect the microenvironment of tumor and hence contribute to survival prediction in colon cancer.

Meanwhile, our results were in accordance with previous specific insights(Koi \& Carethers

Peer) reviewing PDF | (2020:06:50322:1:1:NEW 11 Sep 2020) 
251 2017; Zhang et al. 2018), and might provide extra data in the mining of interaction between 252 tumor and environment in colon cancer.

253 Then, we screened out 572 microenvironment-related DEGs, and found they were mainly 254 enriched in plasma membrane (CC), receptor activity (MF), immune response (BP), and 255 epithelial-to-mesenchymal transition (pathway). Afterwards, AQP4, ASTN1, ATP2B3, CADM3, 256 CD22, CD37, CD79B, CLVS2, CXCR5, FCRLA, GABRG2, MS4A1, NRXN1, SNAP25, and 257 SYT4 were identified as prognostic hub genes, and two of them were verified to be prognosis 258 biomarkers in GEO database. qRT-PCR results revealed that AQP4 and SNAP25 were 259 significantly elevated in colon cancer tissues compared with adjacent normal tissues $(\mathrm{P}=0.003$, 260 0.001). Next, we investigated the underlying mechanism of AQP4 and SNAP25 in colon cancer 261 by GSEA, and found that the high expression of SNAP25 might be involved in cancer-related 262 signaling pathway, immunity and metabolism processes. Further researches in TISIDB database 263 indicated greatest positive correlations between SNAP25 expression and TILs (NK, NKT, 264 macrophage, mast), and greatest negative correlations between SNAP25 methylation and TILs 265 (pDC, Th1, Tfh, NKT). TILs are associated with prognosis for the survival of various tumors in 266 many previous studies. TILs are also reported as a predictive biomarker in colon cancer(Zhao et 267 al. 2019). However, some studies found no significant association between TILs and overall 268 survival. It remains controversial on the prognostic value of TILs in colon cancer may be due to 269 different TIL responses or subsets, different patient populations, different study designs, and the 270 heterogeneity of immune infiltrate. Further studies should be designed to provide more insight 271 into the study of TILs and survival.

272 SNAP25 belongs to the soluble N-ethylmaleimide-sensitive factor attachment protein 273 receptor (SNARE) complex, which is essential for neurotransmitter release, synaptic, secretory 274 vesicle exocytosis, minimal fusion machinery, cell-to-cell signaling, and the regulation of ion 275 channels(Baker \& Hughson 2016; Wang et al. 2017; Yoon \& Munson 2018). As reported, 276 SNAP25 is potentially important for normal vesicle fusion and lysosomal trafficking(Manca et 277 al. 2014; Mu et al. 2018). Kyoko Kobayashi et al(Kobayashi et al. 2016) found that SNAP25 278 protein was expressed in 46\% (77/168) of diffuse large B-cell lymphoma (DLBCL) patients and 279 associated with $\mathrm{CD} 5$ expression $(\mathrm{P}=0.018)$. Huang $\mathrm{CJ}$ et al(Huang et al. 2017) displayed the 280 expression and functional significance of SNAP25 in medulloblastoma. 
Although the clinical significances and functions of SNAP25 for colon cancer have not

282 been previously reported, it may serve as prognosis biomarker according to this study. To our 283 greatest interest, SNAP25 is the most highly interconnected nodes (Figure 4A), and involves in

284

285 286

287 288 289

290

291

292

293

294

295

296

297

298

299

300

301

302

303

304

305

306

307

308

309

310

311 many cancer-related processes consists of adherens junction, calcium signaling pathway, cytokine receptor interaction, ECM receptor interaction, hedgehog signaling pathway, JAKSTAT signaling pathway, MAPK signaling pathway, TGF beta signaling pathway, Toll-like receptor signaling pathway, VEGF signaling pathway, and WNT signaling pathway (Figure 7). Besides, SNAP25 is also involved in immunity and metabolism processes such as B cell receptor signaling pathway, cell adhesion molecules cams, chemokine signaling pathway, complement and coagulation cascades, $\mathrm{T}$ cell receptor signaling pathway, adipocytokine signaling pathway, aldosterone regulated sodium reabsorption, glycosaminoglycan biosynthesis heparan sulfate and insulin signaling pathway. Those may bring novel insights into the potential underlying mechanism of SNAP25 in colon cancer. In addition, this paper reveals the significant correlations between SNAP25 and lymphocytes (NK, macrophage, mast cell, NKT), which indicates the potential association of tumor microenvironment and SNAP25. Recently, a lot of attention has been paid to tumor microenvironment and immune evasion for future diagnoses and treatments of malignant tumors. Immunotherapies have been revolutionizing tumor treatment, although immunological response in different patients is heterogeneous. NK cells participate in tumor immunosurveillance, and are one of the most promising therapies for various types of cancer. However, NK cell populations may shape with altered reactivity in malignant tumors(Hofer \& Koehl 2017). In colorectal cancer, a high level of mast cells was confirmed with poor survival, and the density of innate immune cells (macrophages, mast cells, neutrophils, and immature dendritic cells) increased with tumor stage(Koi \& Carethers 2017). Therefore, SNAP25 is extremely closely associated with various types of TILs, and has the potential to serve as a prognosis biomarker and an immunotherapeutic target for colon cancer.

However, our study presents a number of limitations. One major limitation is that the present research was mainly based on previous data from TCGA and GEO, therefore, future investigations in vivo and vitro are needed to investigate the effect of SNAP25 in colon cancer. A second issue is that since the integrated bioinformatics analysis was focus on immune microenvironment for colon cancer, and the DEGs were identified based on immune and stromal scores, there was no validation for these DEGs based on control samples. Finally, we are lacking 
312 of the specimens of colon cancer and adjacent normal tissues. So, we only validate the

313 expressions of AQP4 and SNAP25 in 20 pairs of tissues by qRT-PCR.

\section{Conclusions}

315 In summary, SNAP25 is a microenvironment-related and immune-related gene that can

316 predict poor outcomes in colon cancer. Bioinformatic analysis suggests that SNAP25 is involved

317 in cancer-related signaling pathway, immunity and metabolism processes, which may provide a

318 new target for investigating the underling mechanism of colon cancer.

319

320

321

322

323

\section{Acknowledgements}

This work was supported by one grant from Health and Family planning Commission of Zhejiang Province (2020KY1045) and one grant from Taizhou Science and Technology Bureau in Zhejiang Province (1901ky62). And the authors would like to thank Qingqing Xia (Central laboratory of Taizhou First People's Hospital) for her technical assistance in qRT-PCR.

\section{References}

Baker RW, and Hughson FM. 2016. Chaperoning SNARE assembly and disassembly. Nat Rev Mol Cell Biol 17:465-479.

Barros MR, Jr., de Melo CML, Barros MLCMGR, de Cássia Pereira de Lima R, de Freitas AC, and Venuti A. 2018. Activities of stromal and immune cells in HPV-related cancers. Journal of experimental \& clinical cancer research : $C R$ 37:137-137.

Garcia-Gomez A, Rodríguez-Ubreva J, and Ballestar E. 2018. Epigenetic interplay between immune, stromal and cancer cells in the tumor microenvironment. Clinical immunology (Orlando, Fla) 196:64-71.

Ghesquière B, Wong BW, Kuchnio A, and Carmeliet P. 2014. Metabolism of stromal and immune cells in health and disease. Nature 511:167-176.

Hofer E, and Koehl U. 2017. Natural Killer Cell-Based Cancer Immunotherapies: From Immune Evasion to Promising Targeted Cellular Therapies. Frontiers in immunology 8:745-745.

Huang CJ, Lee CL, Liu CY, Huang SH, Hou JW, Chen YH, Chien CC, Ho CM, Lo WC, and Hung KL. 2017. Detection of lower levels of SNAP25 using multiple microarray systems and its functional significance in medulloblastoma. Int J Mol Med 39:1195-1205.

Jia D, Li S, Li D, Xue H, Yang D, and Liu Y. 2018. Mining TCGA database for genes of prognostic value in glioblastoma microenvironment. Aging 10:592-605.

Kobayashi K, Yamaguchi M, Miyazaki K, Imai H, Yokoe K, Ono R, Nosaka T, and Katayama N. 2016. Expressions of SH3BP5, LMO3, and SNAP25 in diffuse large B-cell lymphoma cells and their association with clinical features. Cancer Med 5:1802-1809.

Koi M, and Carethers JM. 2017. The colorectal cancer immune microenvironment and approach to immunotherapies. Future oncology (London, England) 13:1633-1647.

Manca P, Mameli O, Caria MA, Torrejon-Escribano B, and Blasi J. 2014. Distribution of SNAP25, VAMP1 and VAMP2 in mature and developing deep cerebellar nuclei after estrogen administration. Neuroscience 266:102-115.

Mu Y, Yan X, Li D, Zhao D, Wang L, Wang X, Gao D, Yang J, Zhang H, Li Y, Sun Y, Wei Y, Zhang Z, Chang X, Yao Z, Tian S, Zhang K, Terada LS, Ma Z, and Liu Z. 2018. NUPR1 maintains autolysosomal efflux by activating SNAP25 transcription in cancer cells. Autophagy 14:654-670.

Nilendu P, Sarode SC, Jahagirdar D, Tandon I, Patil S, Sarode GS, Pal JK, and Sharma NK. 2018. Mutual concessions and compromises between stromal cells and cancer cells: driving tumor development and drug resistance. Cellular oncology (Dordrecht) 41:353-367.

Peer] reviewing PDF | (2020:06:50322:1:1:NEW 11 Sep 2020) 
Petitprez F, Vano YA, Becht E, Giraldo NA, de Reyniès A, Sautès-Fridman C, and Fridman WH. 2018. Transcriptomic analysis of the tumor microenvironment to guide prognosis and immunotherapies. Cancer Immunology, Immunotherapy 67:981-988.

Ru B, Wong CN, Tong Y, Zhong JY, Zhong SSW, Wu WC, Chu KC, Wong CY, Lau CY, Chen I, Chan NW, and Zhang JW. 2019. TISIDB: an integrated repository portal for tumor-immune system interactions. Bioinformatics 35:4200-4202.

Siegel RL, Miller KD, Fedewa SA, Ahnen DJ, Meester RGS, Barzi A, and Jemal A. 2017. Colorectal cancer statistics, 2017. CA Cancer J Clin 67:177-193.

Siegel RL, Miller KD, and Jemal A. 2020. Cancer statistics, 2020. CA Cancer J Clin 70:7-30.

Wang H, Wu X, and Chen Y. 2019. Stromal-Immune Score-Based Gene Signature: A Prognosis Stratification Tool in Gastric Cancer. Frontiers in Oncology 9.

Wang T, Li L, and Hong W. 2017. SNARE proteins in membrane trafficking. Traffic 18:767-775.

Wu T, and Dai Y. 2017. Tumor microenvironment and therapeutic response. Cancer letters 387:61-68.

Yan H, Qu J, Cao W, Liu Y, Zheng G, Zhang E, and Cai Z. 2019. Identification of prognostic genes in the acute myeloid leukemia immune microenvironment based on TCGA data analysis. Cancer Immunol Immunother 68:1971-1978.

Yoon TY, and Munson M. 2018. SNARE complex assembly and disassembly. Curr Biol 28:R397-R401.

Yoshihara K, Shahmoradgoli M, Martinez E, Vegesna R, Kim H, Torres-Garcia W, Trevino V, Shen H, Laird PW, Levine DA, Carter SL, Getz G, Stemke-Hale K, Mills GB, and Verhaak RG. 2013. Inferring tumour purity and stromal and immune cell admixture from expression data. Nat Commun 4:2612.

Zhan H-X, Zhou B, Cheng Y-G, Xu J-W, Wang L, Zhang G-Y, and Hu S-Y. 2017. Crosstalk between stromal cells and cancer cells in pancreatic cancer: New insights into stromal biology. Cancer letters 392:83-93.

Zhang M, Pang H-J, Zhao W, Li Y-F, Yan L-X, Dong Z-Y, and He X-F. 2018. VISTA expression associated with CD8 confers a favorable immune microenvironment and better overall survival in hepatocellular carcinoma. BMC Cancer 18.

Zhao Y, Ge X, He J, Cheng Y, Wang Z, Wang J, and Sun L. 2019. The prognostic value of tumor-infiltrating lymphocytes in colorectal cancer differs by anatomical subsite: a systematic review and meta-analysis. World J Surg Oncol 17:85. 


\section{Figure 1}

Figure 1. Stromal and immune scores are associated with the clinical features of colon cancer and their overall survival.

(A) Distribution of stromal scores of colon cancer between different histologic diagnosis. (B) The correlation between stromal scores and anatomic neoplasm subdivision. (C) Distribution of immune scores of colon cancer between different histologic diagnosis. (D) The correlation between immune scores and anatomic neoplasm subdivision. (E) Kaplan-Meier survival curve for patients with low vs. high stromal scores. (F) Kaplan-Meier survival curve for patients with low vs. high immune scores. (G) Kaplan-Meier survival curve for patients with low vs. high both stromal and immune scores. 

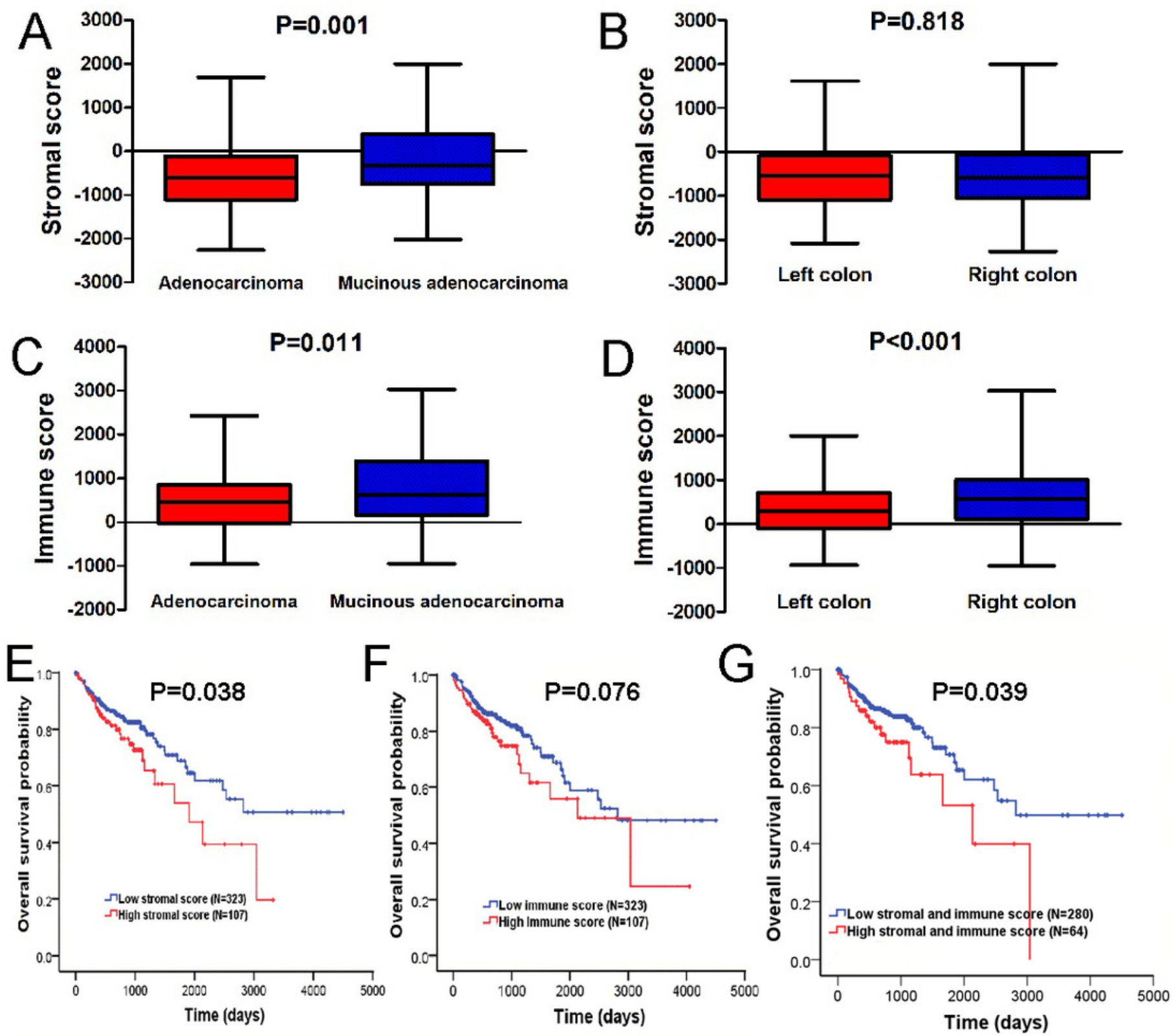


\section{Figure 2}

Figure 2. Expression profiles and biological functions of DEGs based on stromal and immune scores.

(A) Volcano plot showing up-regulated DEGs in red and down-regulated DEGs in blue for the comparison based on high and low stromal score groups. (B) Volcano plot showing upregulated DEGs in red and down-regulated DEGs in green for the comparison based on high and low immune score groups. (C, D) Venn diagrams showing 563 shared up-regulated DEGs (C) and 9 shared down-regulated DEGs (D) from stromal score and immune score groups. (E, F, G, H) Top six Go terms and pathways enriched by DEGs. 


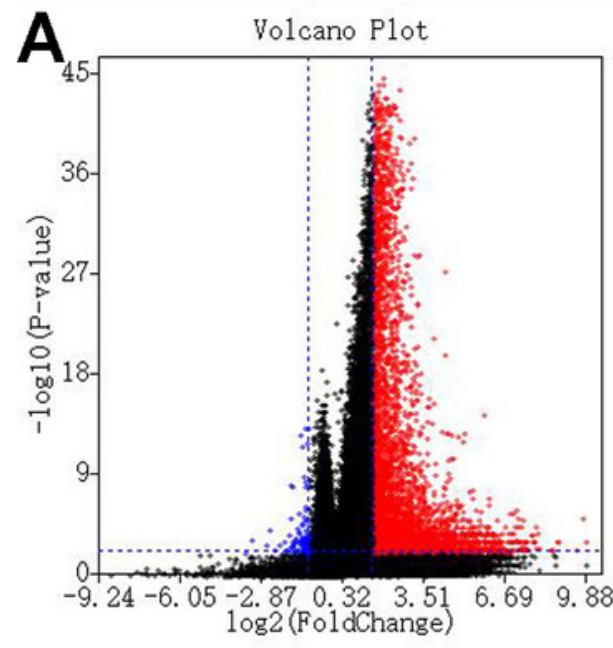

Stromal score
B

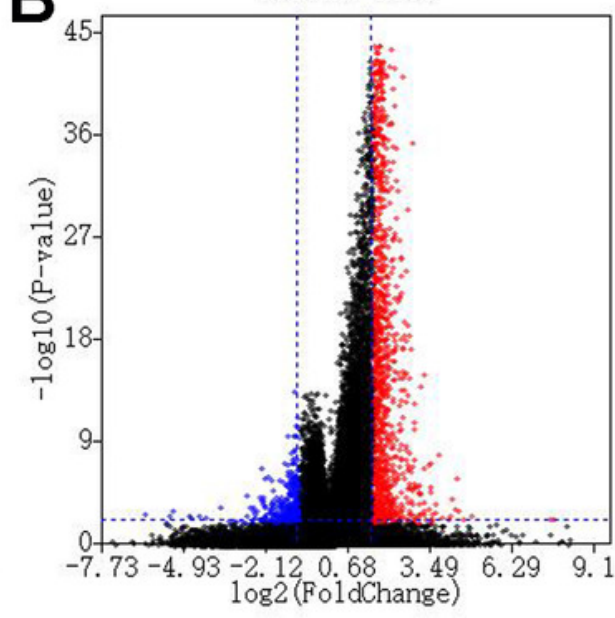

C Up-regulated DEGs

Stromal score Immune score

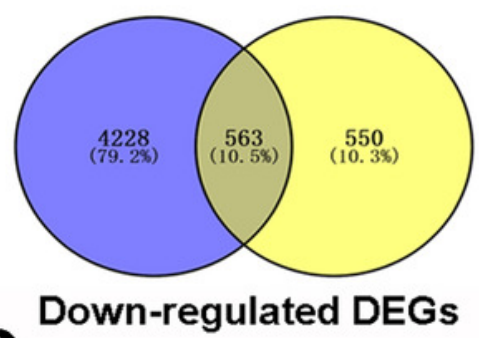

D
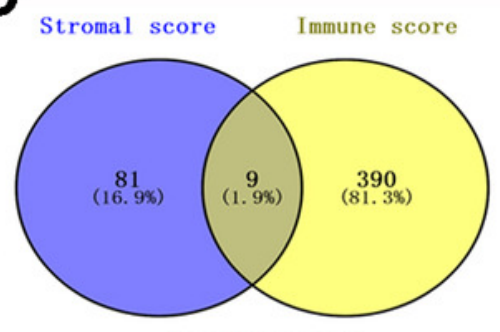

- Log10 (p-value)

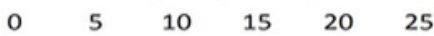

Growth factor activity Chemokine activity Extracellular matrix structural...

$B$ cell receptor activity Cell adhesion molecule activity Receptor activity

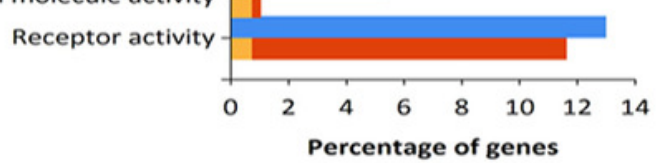

H

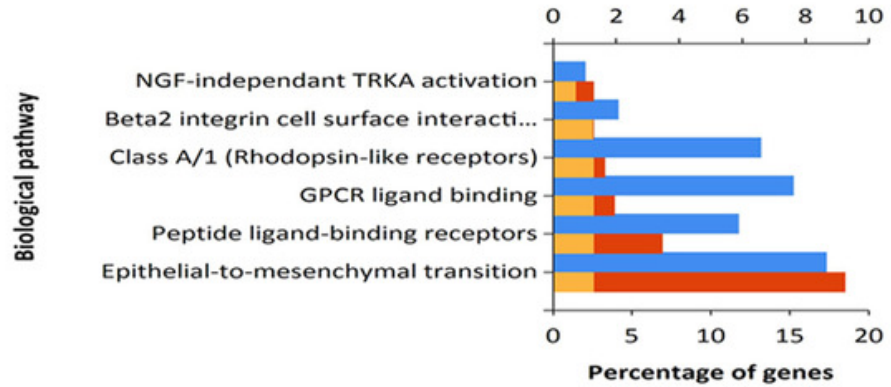


Figure 3

Figure 3. Forest plot of hazard ratios (HR) for 70 prognostic DEGs in colon cancer. HR and $95 \%$ confidence intervals $(\mathrm{Cl})$ were obtained by the COX proportional hazards model.

HR and $95 \%$ confidence intervals (Cl) were obtained by the COX proportional hazards model.
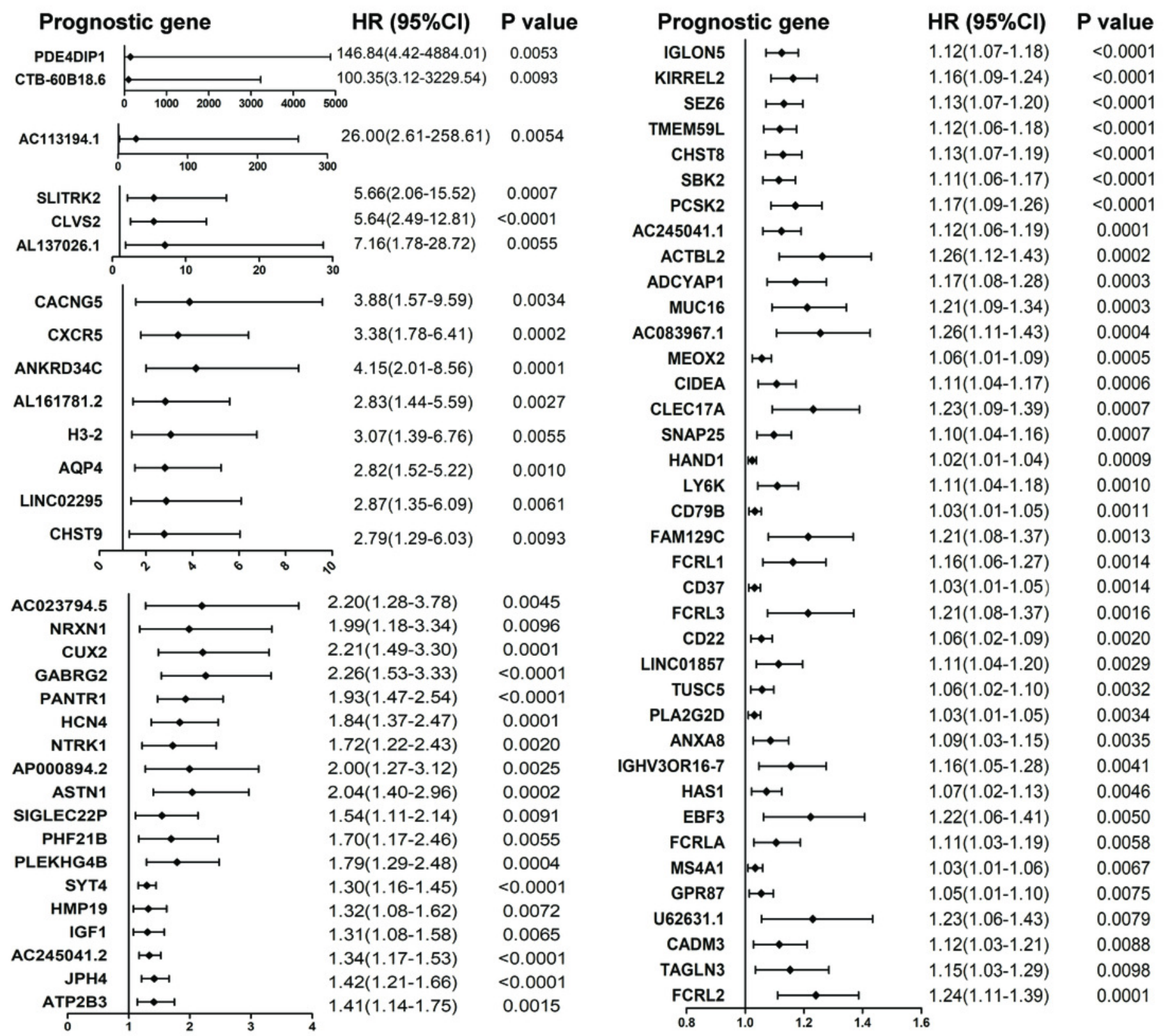


\section{Figure 4}

Figure 4. PPI network, GO analysis and expression of hub gene.

(A) PPI network contained 30 nodes and 53 edges based on STRING tool and Cytoscape software was constructed. The hub genes were selected by MCODE in yellow with 9 nodes and 22 edges in the left module, and 6 nodes and 12 edges in the right module. (B) The GO analysis of hub genes was performed using BiNGO. The color of the node represented the corrected P-value of ontologies, and the size of the node represented the numbers of involved genes in the ontologies. $P<0.01$ was considered statistically significant. (C) The expression of 15 hub genes in TCGA cohort by z-score, with red represents higher expression and blue represents lower expression. 


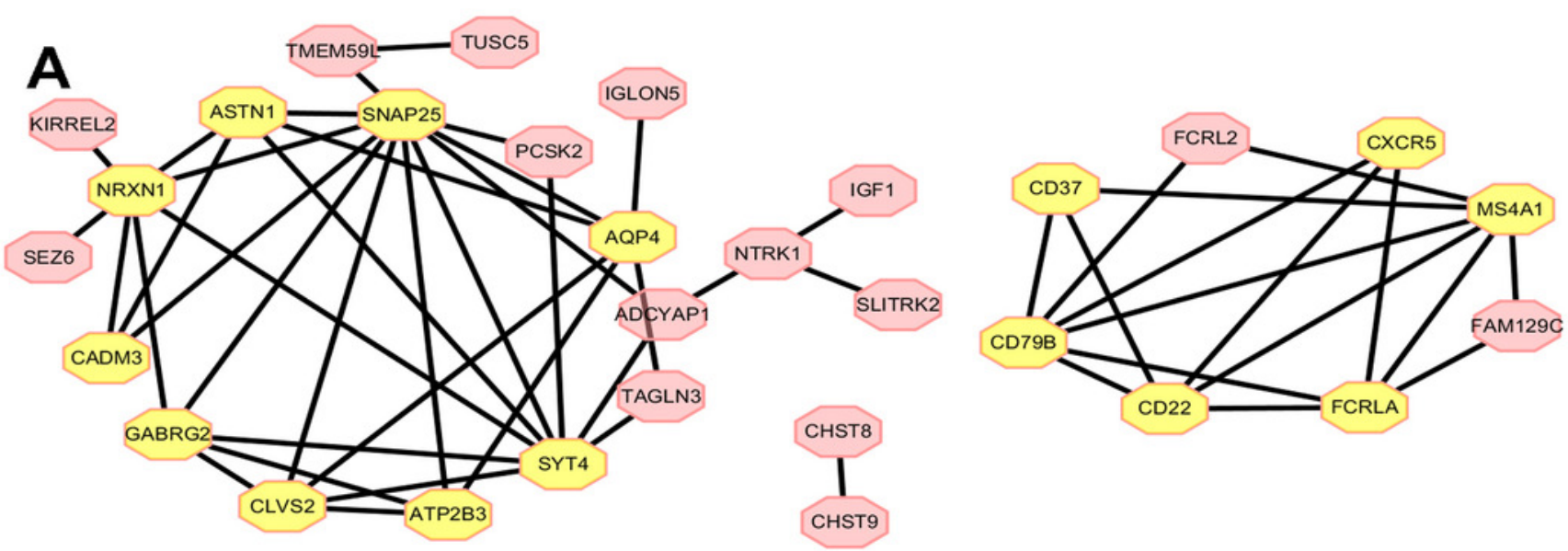

B

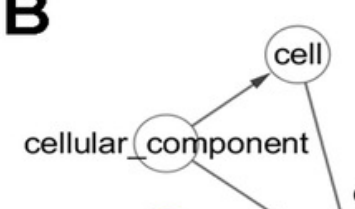

external side of plasma membrane integral to plasma
membrane transmembrane
transporter activity
transporteractivity
transporter activity cell surface
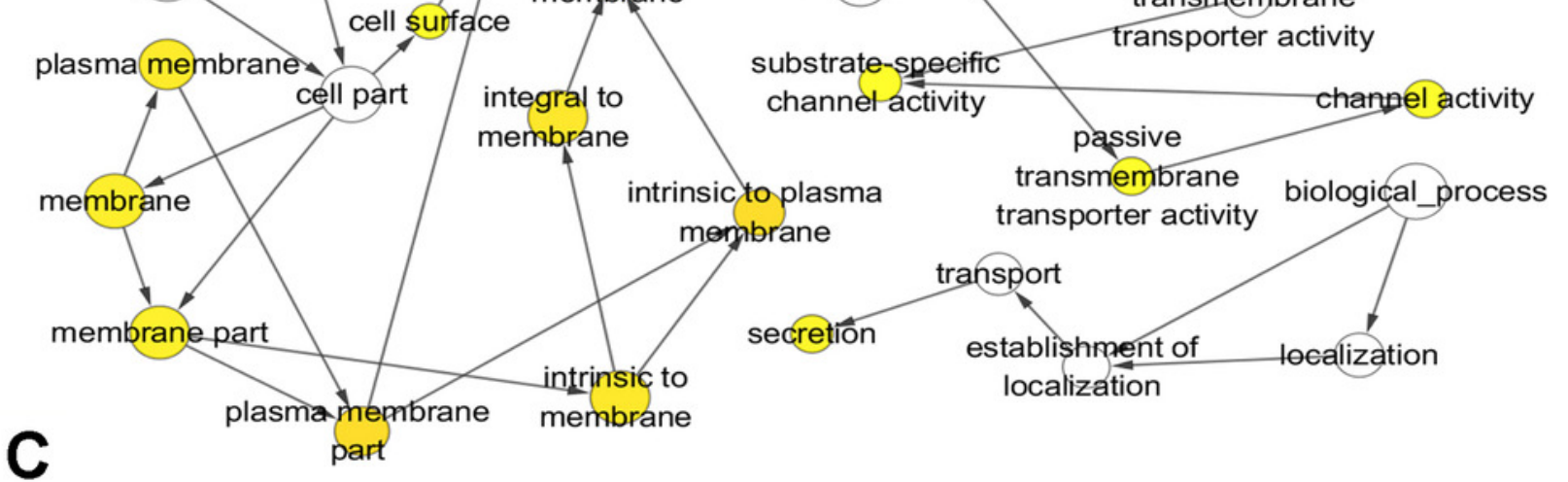

molecular_function

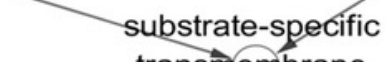
substrate-speeific transmembrane

Zscore Cene Empression

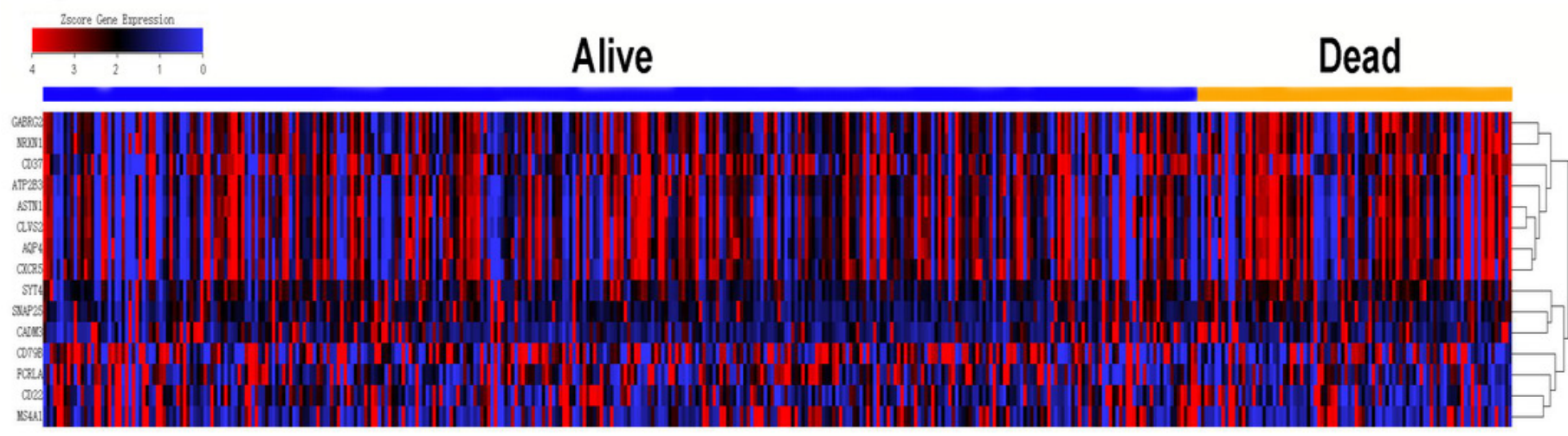




\section{Figure 5}

Figure 5. verifying the survival outcomes of hub genes in the GEO database.

Kaplan-Meier survival curves with the log-rank test and COX proportional hazards regression analysis were generated for prognostic genes. The dotted blue/ red lines indicated 95\% confidence intervals of overall survival probability. $\mathrm{P}<0.05$ was considered statistically significant.
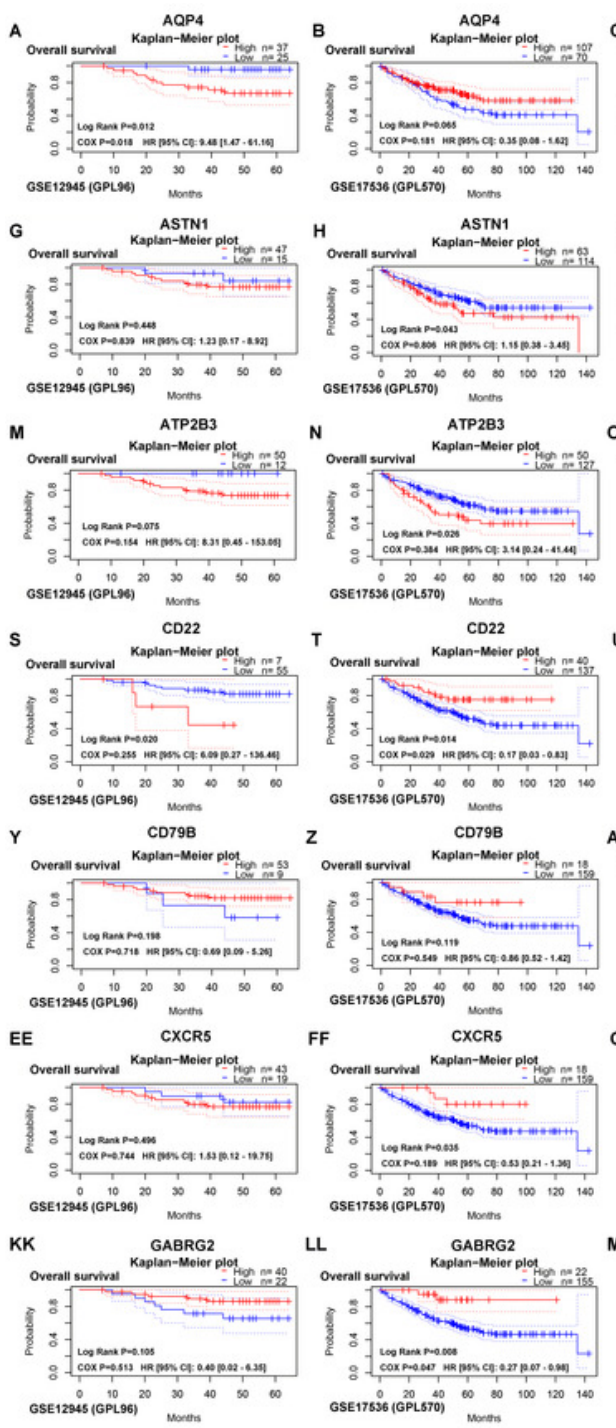

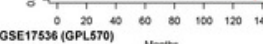

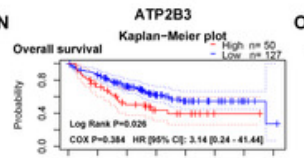

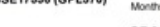

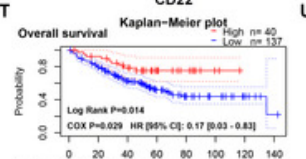
$\begin{array}{cccccc}0.00 & 10 & 1 & 1 & 1 & 1 \\ 0 & 00 & 100 & 120 & 10\end{array}$
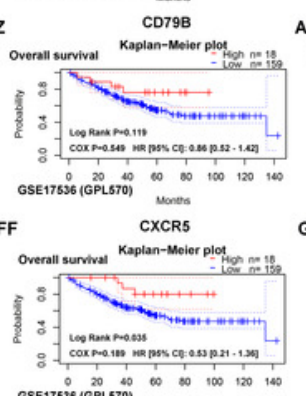

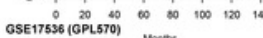
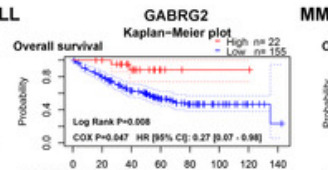

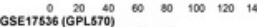
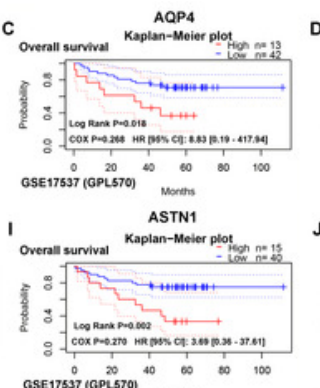

ATP2B3
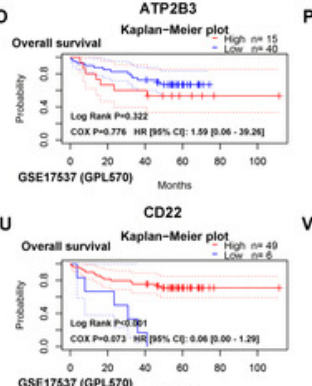

AA
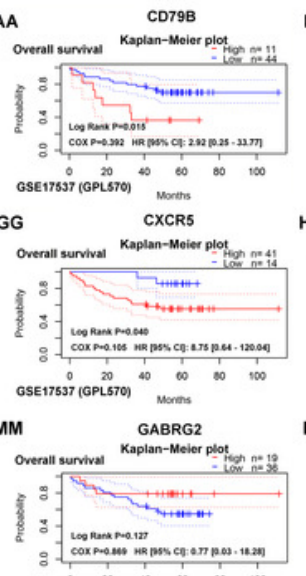
GSE 17537 (GPLSTO) 40 )
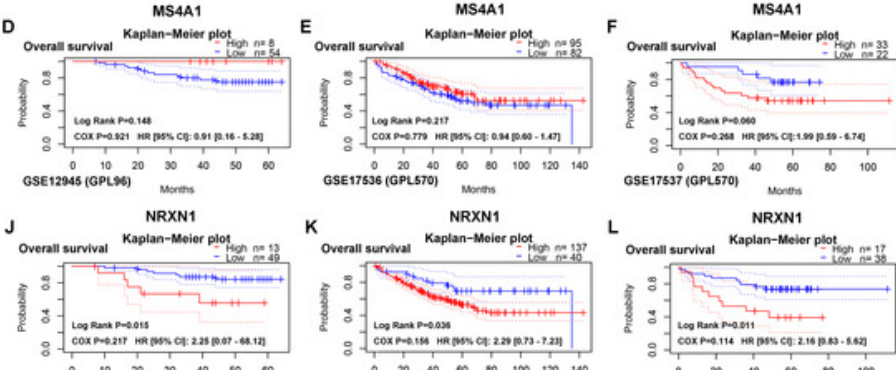

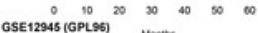

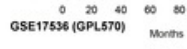
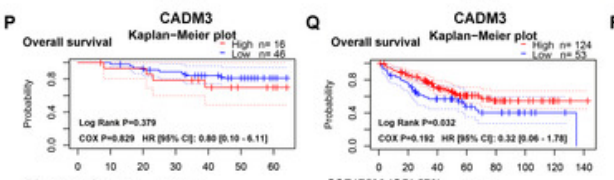
GSE12945 (GPLS) Whet

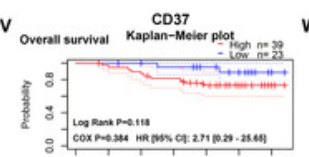

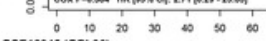
BB
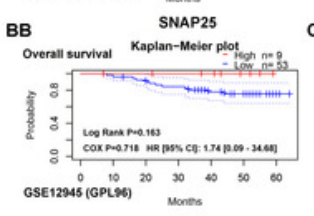

$\mathrm{HH}$
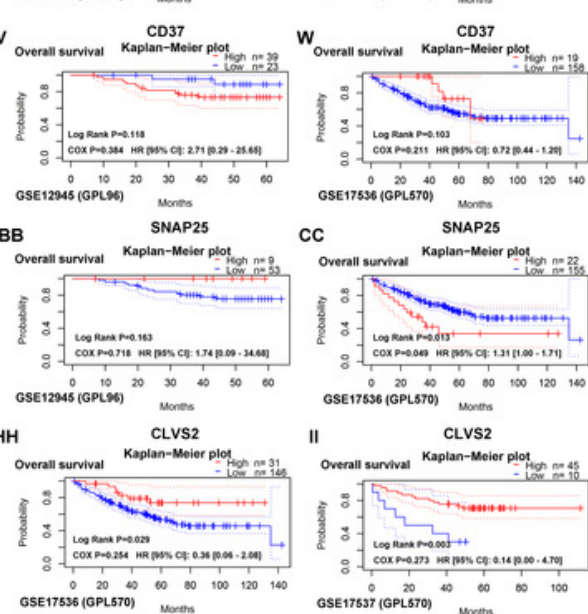

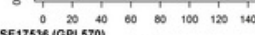
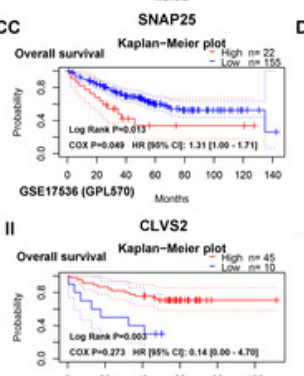
csetras?
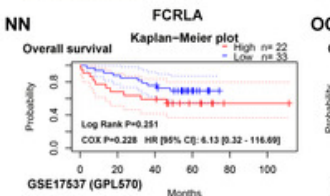

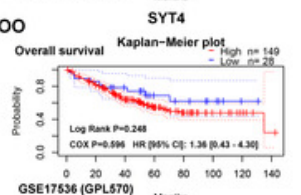

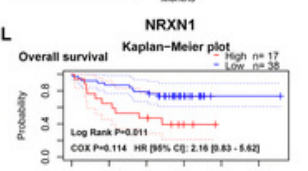
$\underset{\text { GSE17537 (GPLSTO) }}{20}{ }^{40} \quad 60 \quad 50 \quad 100$
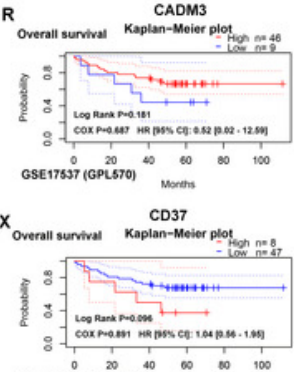

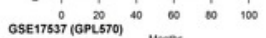
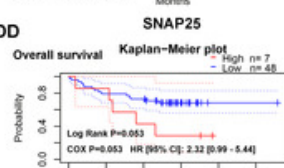

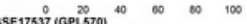

J)

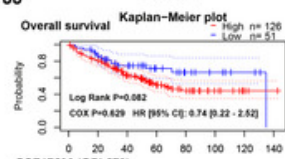
GSE175S6 (GPLSTO)
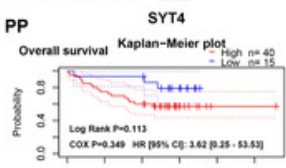
GSE17037 (GPLSTO) ${ }^{20}$ 10 $10 \quad 80100$ GSE17537 (GPLSTO) 
Figure 6

Figure 6 The relative mRNA expression levels of AQP4 (A) and SNAP25 (B) in colon cancer tissues and adjacent normal tissues were confirmed by qRT-PCR.
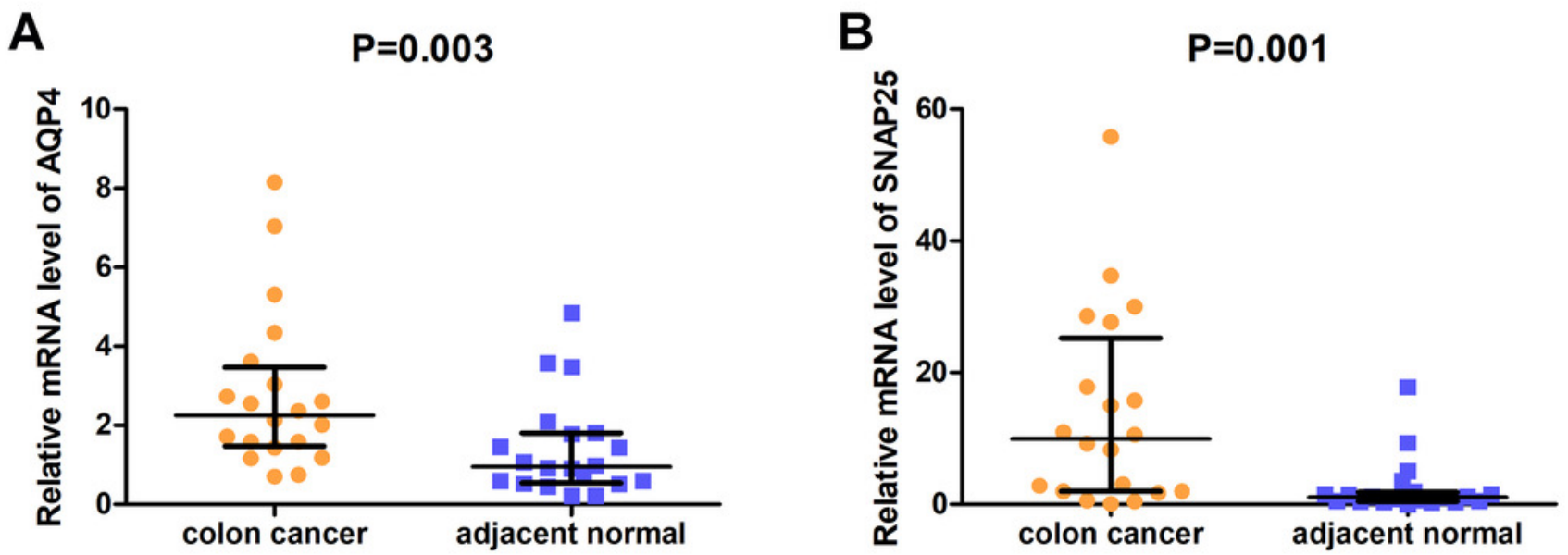
Figure 7

Figure 7. GSEA enrichment plots showed that eleven gene sets related to tumor signaling pathways were enriched in the high SNAP25 expression group. 


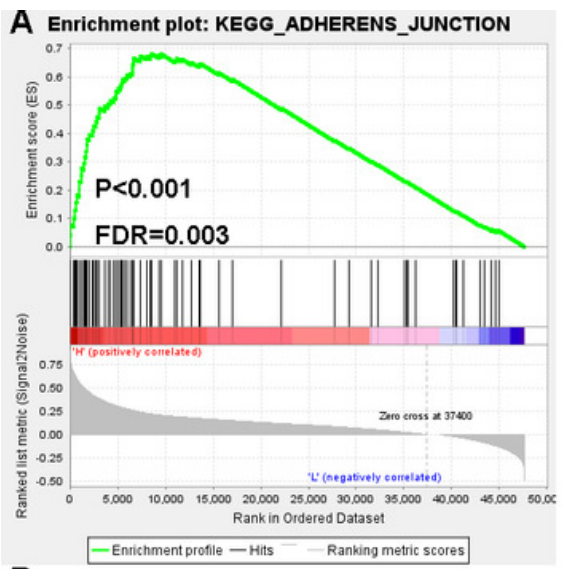

D

Enrichment plot: KEGG_ECM_RECEPTOR_INTERACTION

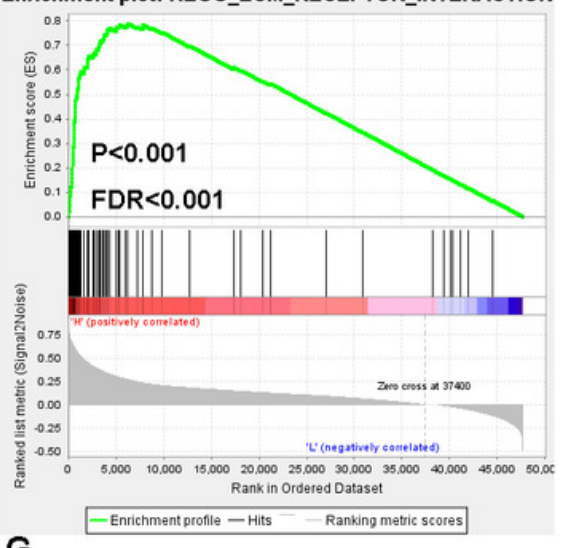

Enrichment plot: KEGG_MAPK_SIGNALING_PATHWAY

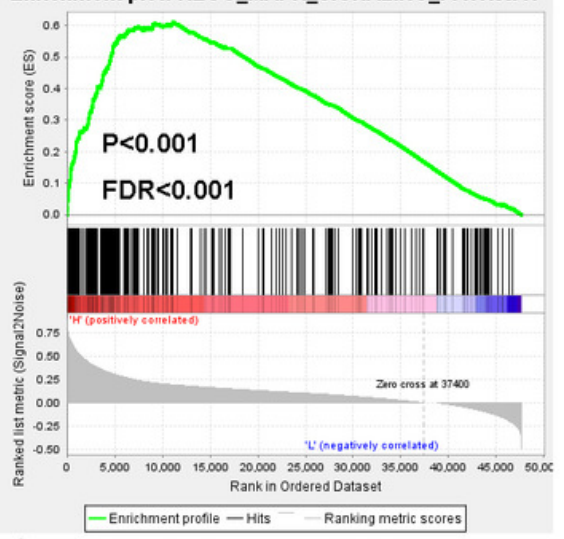

J Enrichment plot: KEGG_VEGF_SIGNALING_PATHWAY

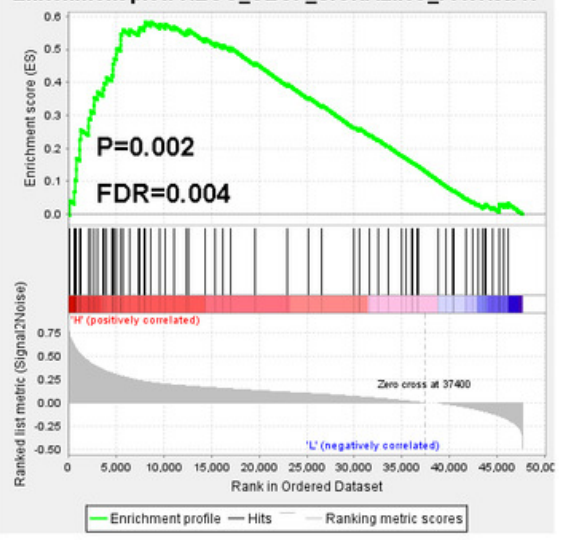

B

B Enrichment plot: KEGG_CALCIUM_SIGNALING_PATHWAY

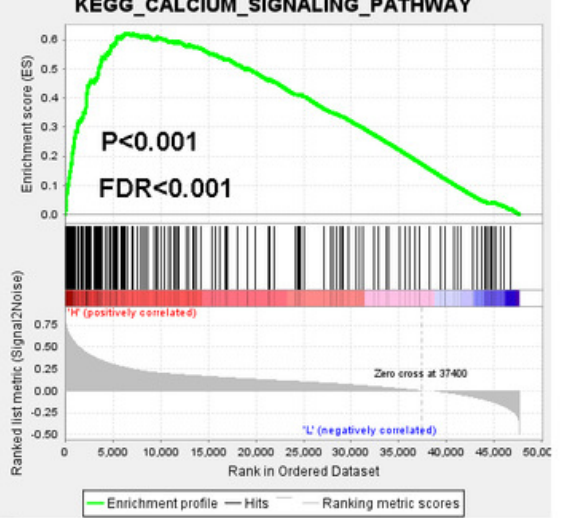

E

Enrichment plot:

KEGG_HEDGEHOG_SIGNALING_PATHWAY

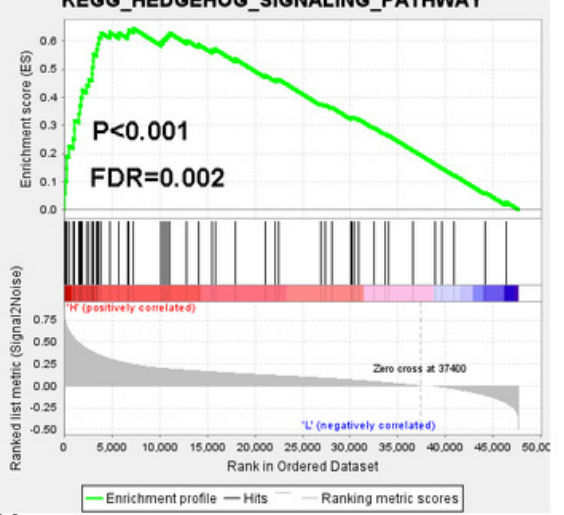

H

Enrichment plot:

KEGG_TGF_BETA_SIGNALING_PATHWAY
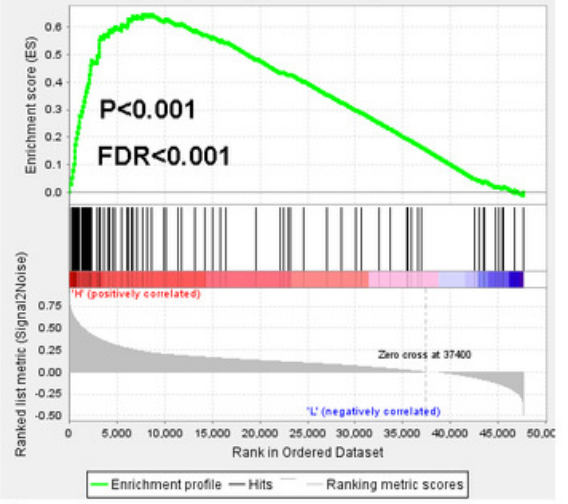

K Enrichment plot: KEGG_WNT_SIGNALING_PATHWAY

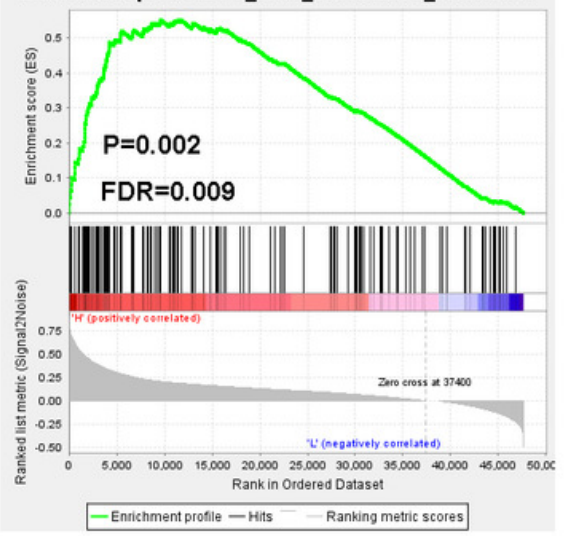

C Enrichment plot:

KEGG_CYTOKINE_CYTOKINE_RECEPTOR_INTERACTIO

N

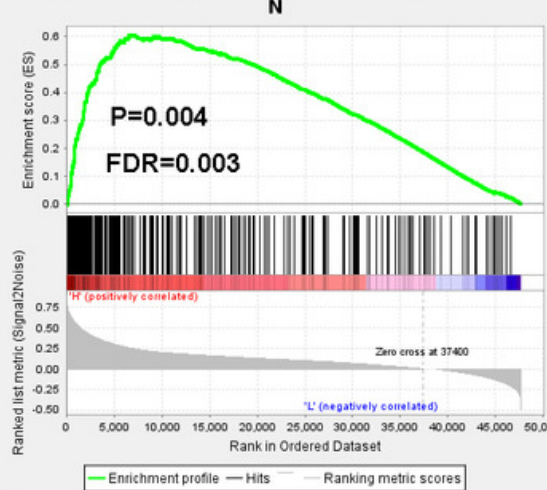

$\mathbf{F}$

Enrichment plot:

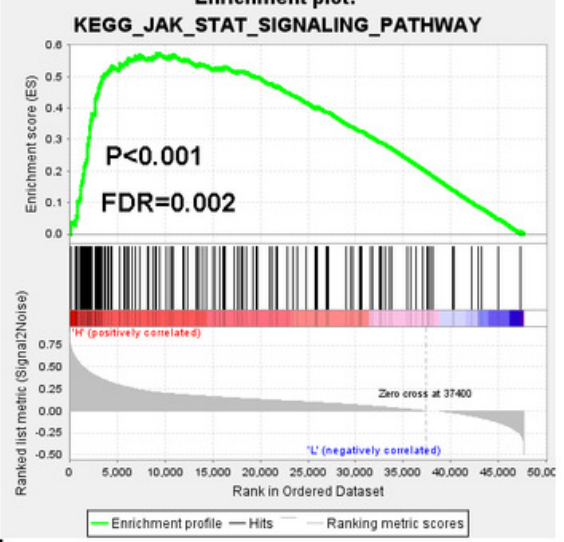

I
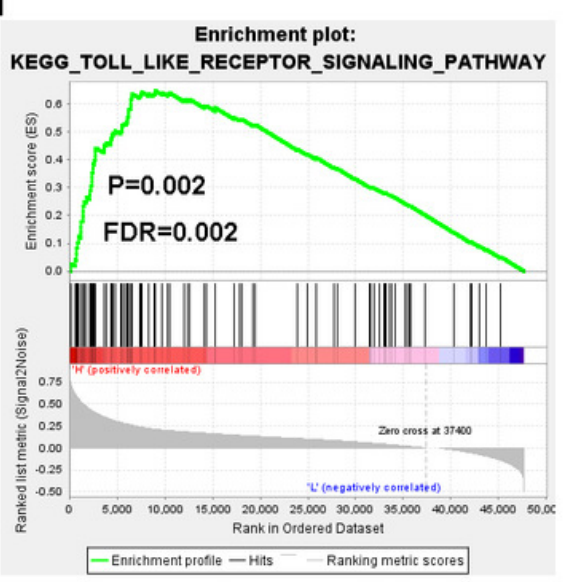


\section{Figure 8}

Figure 8. Spearman's correlations between SNAP25 and lymphocytes (TISIDB).

(A) Relations between the SNAP25 expression and abundance of TILs across human cancers.

(B) Top 4 greatest positive correlations between SNAP25 expression and TILs. (C) Relations between the SNAP25 methylation and abundance of TILs across human cancers. (D) Top 4 greatest negative correlations between SNAP25 methylation and TILs. 

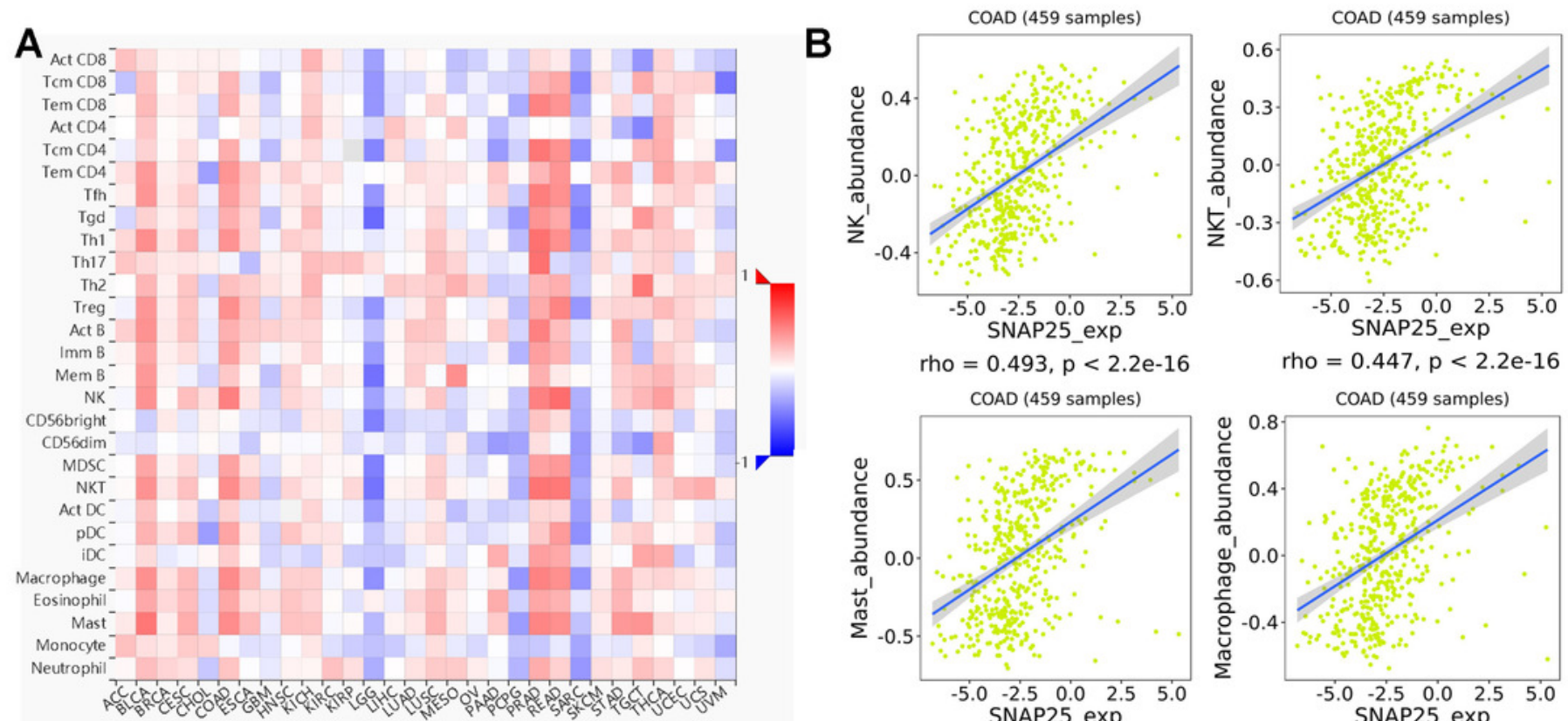

rho $=0.493, \mathrm{p}<2.2 \mathrm{e}-16$

rho $=0.447, p<2.2 \mathrm{e}-16$
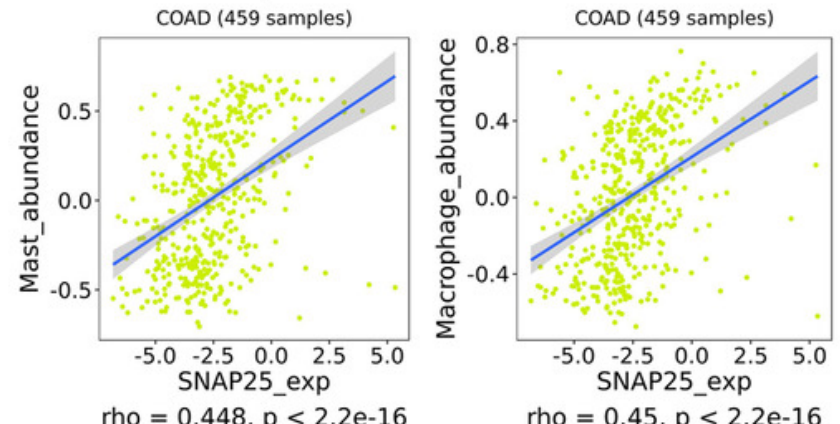

ho $=0.448, p<2.2 \mathrm{e}-16$

C

$\mathrm{C}_{\mathrm{ACCCB}}$ ${ }_{\operatorname{Tem} C D 8^{-}}^{\operatorname{Trm} C D^{-}}$

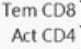

$\mathrm{Tcm} \mathrm{CD}_{4}^{-}$

Tem $\mathrm{CD}_{4}{ }^{-}$

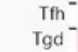

$\mathrm{Tgd}^{-}$
$\mathrm{Th}^{-}$
$\mathrm{Th1} 7^{-}$
$\mathrm{Th}^{-}$

Th2
Treg
Act B

Act B
Imm B

$\operatorname{Imm} B^{-}$
$\mathrm{MemB} B^{-}$
$\mathrm{NK}^{-}$

CD56bright

$\mathrm{CDS}_{6} \mathrm{dim}$
$\mathrm{MDSC}$

MDSC
NKT

Act DC -

$\mathrm{PDC}^{-}$

Macrophage -

Eosinophil

Mast ${ }^{-}$

Neutrophil

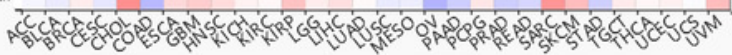
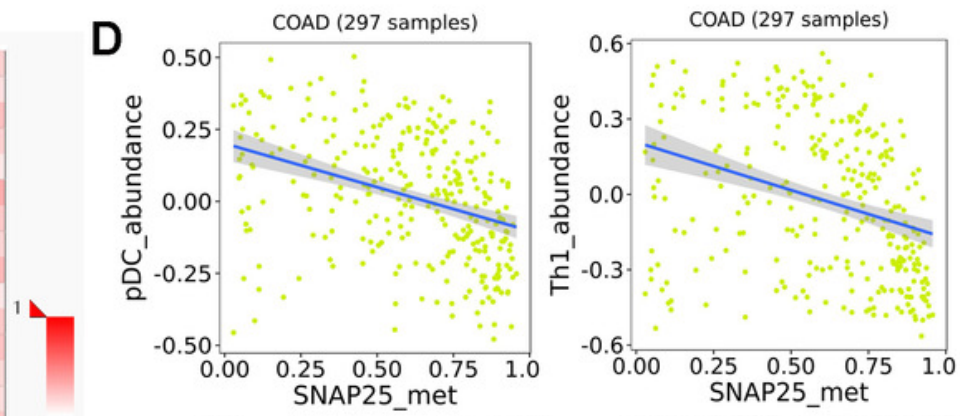

rho $=-0.419, p=2.96 \mathrm{e}-14$

rho $=-0.406, p=4.06 e-13$
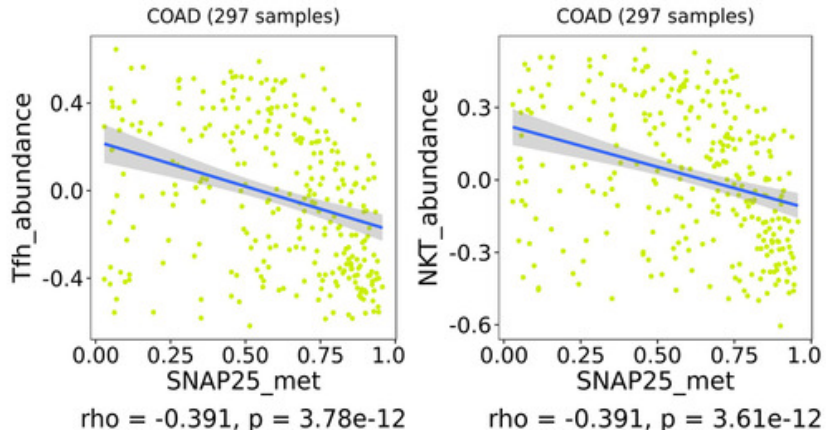